\title{
Stability and Identification with Optimal Macroprudential Policy Rules*
}

\author{
Jean-Bernard Chatelain ${ }^{\dagger}$ and Kirsten Ralf ${ }^{\ddagger}$
}

November 9, 2018

\begin{abstract}
This paper investigates the identification, the determinacy and the stability of ad hoc, "quasi-optimal" and optimal policy rules augmented with financial stability indicators (such as asset prices deviations from their fundamental values) and minimizing the volatility of the policy interest rates, when the central bank precommits to financial stability. Firstly, ad hoc and quasi-optimal rules parameters of financial stability indicators cannot be identified. For those rules, non zero policy rule parameters of financial stability indicators are observationally equivalent to rule parameters set to zero in another rule, so that they are unable to inform monetary policy. Secondly, under controllability conditions, optimal policy rules parameters of financial stability indicators can all be identified, along with a bounded solution stabilizing an unstable economy as in Woodford (2003), with determinacy of the initial conditions of non- predetermined variables.

JEL classification numbers: C61, C62, E43, E44, E47, E52, E58.
\end{abstract}

${ }^{*}$ We thank for helpful comments Antoine d'Autume, Hippolyte d'Albis, Gunther Capelle-Blancard, Robert Chirinko, Jean-Pierre Drugeon, Pedro Garcia Duarte, Roger Farmer, Stephane Gauthier, Mauro Napoletano, Michel de Vroey and Bertrand Wigniolle, as well as participants in the Macroeconomics in Perspective Workshop in Louvain la Neuve and the seminar "Dynamique de la macroéconomie" in Paris 1 Pantheon Sorbonne.

${ }^{\dagger}$ Paris School of Economics, Université Paris I Pantheon Sorbonne, CES, Centre d'Economie de la Sorbonne, 106-112 Boulevard de l'Hôpital 75647 Paris Cedex 13. Email: jean-bernard.chatelain@univ-paris1.fr

${ }^{\ddagger}$ ESCE International Business School, 10 rue Sextius Michel, 75015 Paris, Email: Kirsten.Ralf@esce.fr. 
Keywords: Identification, Financial Stability, Optimal Policy under Commitment, Augmented Taylor rule, Monetary Policy.

"If we ran the Taylor rule regression in data generated by the new-Keynesian model, we would recover the shock autocorrelation process, not the Taylor rule parameter". Cochrane (2011, online appendix, p.15).

"We may omit consideration of the transversality conditions, as we shall consider only bounded solutions to these equations, which necessarily satisfy the transversality conditions." Woodford (2003, p.865).

\section{Introduction}

Should financial stability concerns influence monetary policy decisions? For a policy-maker (Stein (2014)), the argument rests on three assumptions. First, the Federal Reserve cares about minimizing a quadratic loss objective function which includes a "risk" term, given by the variance of realized unemployment, which depend on financial market vulnerability. "Second, there is some variable summarizing financial market vulnerability which is influenced by monetary policy... The third and final assumption is that the risks associated with an elevated value of financial market vulnerability cannot be fully offset at zero cost with other non-monetary tools, such as financial regulation" (Stein (2014), p. 2-3). In this context, the recent macro-prudential dynamic stochastic general equilibrium (DSGE) literature compares the outcomes of ad hoc Taylor rules of the central bank "not augmented" versus "macroprudential rules" augmented with macroprudential indicators such as asset prices and/or households', non-financial firms' and banks' leverage, credit spreads, liquidity ratios and so on (e.g. Beau, Clerc and Mojon (2012), Smets (2013), Chadha, Corrado and Corrado (2013), Gambacorta and Signoretti (2014)).

This paper investigates in a general framework under which conditions the augmented policy rule parameters of financial stability indicators are identified within ad hoc, quasi-optimal or optimal policy rules under commitment to financial stability. It provides complementary results with respect to the lack of identification of non augmented ad hoc Taylor rules parameters found by Cochrane (2011), Komunjer and Ng (2011) and Caglar, Chadha and Shibayama (2012).

With linear quadratic rational expectations optimal rules, the policymaker determines optimal feedback policy rule parameters as a Stackelberg leader in a dynamic game with the private sector. She minimizes a 
quadratic loss function subject to the private sector first order conditions, linearized around an equilibrium (Woodford (2003), Blake and Kirsanova (2012), Ljungqvist and Sargent (2012, chapter 19), Miller and Salmon (1985) among others). DSGE models include $n$ "predetermined" variables with known initial value, such as stationary auto-correlated shocks and capital stocks and $m$ "non-predetermined" variables which are "forward" rational expectations variables with unknown initial values. Examples here are expected inflation, output gap, asset prices, private credit and so on. Levine and Currie (1987) called a policy rule "quasi-optimal" whenever it includes constraints for the parameters of non-predetermined variables to be equal to zero. "Quasi-optimal" policy rules are an intermediate modeling step towards time consistent policy rules (Blake and Kirsanova (2012)).

With quasi-optimal policy rules and ad hoc rules, the policy-maker and the private sector assume transversality conditions (there are no bubbles for non-predetermined variables such as asset prices), seeking Blanchard and Kahn's (1980) unique stable solution. By assumption, it follows that nonpredetermined variables are a linear function (with time invariant coefficients) of predetermined variables for all periods of the model. Then, if one substitutes non-predetermined variables by predetermined variables in a policy rule with rule parameters of non predetermined variables which are not all equal to zero, it leads to another observationally equivalent policy rule with rule parameters for non predetermined variables which are all equal to zero. The parameters of non-predetermined variables cannot be identified in a quasi-optimal or an ad hoc rule. In more technical words, the rank of the dynamic system is equal to the number $n$ of predetermined variables and the eigenvalues related to $m$ "Jordan transformed" non-predetermined variables have been set to zero. Hence, in those models, discussing whether Central Bank policy makers should augment or not Taylor rules with asset prices or financial instability indicators does not inform monetary policy.

If not Blanchard and Kahn's (1980) conditions, then what? This paper proposes sufficient conditions for the identification of rule parameters of non predetermined variables in a general case of Woodford's (2003) and Ljungqvist and Sargent's (2012, chapter 19) optimal policy rules under commitment. These optimal rules are "over stable", according to Levine and Currie's (1987) definition, as the number of stable dimensions of the dynamic system (stable eigenvalues) is equal to $n+m$ the number of variables: it is larger than the number $n$ of predetermined variables. The rank of the dynamic system under control is equal to $n+m$.

Kalman (1960) defined a controllable dynamic system with linear feedback rule when a policy-maker is able to move this dynamic system in any state during any variation of time. A sufficient condition for a controllable system 
is that the policy rule instruments can have an effect on all the ("Jordan" transformed) variables, in particular, all the non-predetermined variables. This is related to Stein's (2014) second assumption: there is some variable summarizing financial market vulnerability which is influenced by monetary policy. In DSGE models, a subset of a system can be checked to be controllable. This subset excludes stationary auto-regressive shocks which are exogenous, hence not controllable.

This paper states that if this subset of the dynamic system of a DSGE is controllable and if all its eigenvalues are distinct and stable, then the linear quadratic regulator optimal policy rule parameters of all controllable variables, including non predetermined variables (more precisely, their predetermined shadow prices), are unique, this set of linear quadratic regulator rule parameters has a one to one correspondence with the set of distinct and stable eigenvalues, and all rule parameters can be identified in optimal policy interest rules under commitment à la Woodford (2003). Indeed, testing optimal rules under commitment against quasi-optimal rules or ad hoc rules is impossible, because the rule parameters of financial instability indicators such as asset prices in "quasi-optimal", ad hoc and time consistent "augmented" Taylor rules cannot be identified, and then cannot be estimable.

The paper then mentions a very important result for the determinacy of New Keynesian models with optimal rules under commitment, which is the opposite of the conventional determinacy criterion for ad hoc rules (Cochrane (2011)). The conventional determinacy criterion is the equality of the number of stable eigenvalues to the number of predetermined variables (Blanchard and Kahn's (1980)). The conventional view leads to the alternative: "bubbles versus sunspots" (Loisel (2009)). New Keynesian macroprudential DSGE ad hoc augmented Taylor rules should not stabilize potential bubbles of $m$ nonpredetermined variables such as asset prices and private credit in order to maintain the uniqueness of a knife-edge stable equilibrium path, knowing that infinitesimal deviations from this path lead to diverging bubbles for the $m$ non-predetermined variables. Else, there will be an infinity of initial values ("sunspots") for the $m$ non-predetermined variables, if ever diverging bubbles are stabilized by Old Keynesian policy-makers rules. However, several economists consider that the knife edge equilibrium is not unique and that an infinity of rational expectations multiple equilibria with diverging paths (bubbles) are also valid (Burmeister (1980), Cochrane (2011), Christiaans (2013)).

Quasi-optimal and time-consistent rules under commitment may face multiple equilibria and indeterminacy when satisfying Blanchard and Kahn's (1980) condition of the equality between the number of stable eigenvalues and the number of predetermined variables (Blake and Kirsanova (2012)). If 
the system is controllable with $n+m$ stable dimensions (stable eigenvalues related to "stable" eigenvectors), there are a number of ways to find $n$ eigenvectors related to stable eigenvalues equal to the number $n$ of predetermined variables, satisfying Blanchard and Kahn's (1980) condition. This number is equal to the number of subsets of $n$ distinct eigenvectors among $n+m$ eigenvectors (Blake and Kirsanova (2012)). For optimal rules under commitment, there is only one subset of $n+m$ distinct eigenvectors of stable eigenvalues among $n+m$ eigenvectors. Finally, if the system is controllable, the Lagrange multipliers of non-predetermined variables with optimal rules under commitment are equal to zero at the initial date, in order to minimize the marginal value of the loss function. This allows to "determine" the unique initial value of each non-predetermined variable, such as financial stability indicators.

Additionally, this paper highlights two useful properties of optimal rules under commitment which inform monetary policy. Empirical literature documents that unexpected changes in the nominal interest rate have a significant effect on real stock prices and on housing prices (Challe and Giannitsarou (2014)). However, Central Bankers fear that stabilizing asset prices and credit bubbles may lead to too much volatility of their interest rate. A first issue faced by policy-makers is then to set a trade-off between the policy rate volatility versus targeting financial stability, understood as leaning against credit and asset price bubbles. This issue cannot be informative with "quasioptimal" rules, as non-predetermined variables cannot be identified.

A second issue is related to the ability of macro-prudential policy to decrease the wealth effect channel of financial instability, i.e. the correlation between asset prices and capital. This issue cannot be addressed with "quasioptimal" rules, because there is a fixed exact linear relationship (and correlation) between non-predetermined variables (asset prices) and predetermined variables (capital) following Blanchard and Kahn (1980) assumption.

This paper proceeds as follows. Section 1 and 2 presents identification of rule parameters with quasi-optimal versus with optimal rules under commitment to financial stability. Section 3 concludes with potential extensions.

\section{Identification in "quasi-optimal" rules with pre-commitment assuming no-bubbles}

Our analysis uses without lack of generality the deterministic setup. The certainty equivalence property of linear quadratic optimal control models implies that optimal rule parameters do not depend from an appropriate 
vector of random shocks which can be added (Anderson et al. (1996), Blake and Kirsanova (2012)). The Central Bank as a Stackelberg leader commits to a sequence of decision rules at time 0 , in a Ramsey problem (Ljundqvist and Sargent (2012), chapter 19). She minimizes her loss function by finding a sequence of decision rules $r_{t}$ :

$$
\begin{gathered}
\max _{\left\{r_{t}, k_{t+1}, q_{t+1}\right\}}-\frac{1}{2} \sum_{t=0}^{+\infty} \beta^{t}\left[\begin{array}{c}
\left(\frac{\mathbf{k}_{t}-\mathbf{k}^{*}}{\mathbf{k}^{*}}\right)^{T} \mathbf{Q}_{n n}\left(\frac{\mathbf{k}_{t}-\mathbf{k}^{*}}{\mathbf{k}^{*}}\right)+\left(\frac{\mathbf{q}_{t}-\mathbf{q}^{*}}{\mathbf{q}^{*}}\right)^{T} \mathbf{Q}_{m m}\left(\frac{\mathbf{q}_{t}-\mathbf{q}^{*}}{\mathbf{q}^{*}}\right) \\
+\left(\frac{\mathbf{k}_{t}-\mathbf{k}^{*}}{\mathbf{k}^{*}}\right)^{T} \mathbf{Q}_{n m}\left(\frac{\mathbf{q} t-\mathbf{q}^{*}}{\mathbf{q}^{*}}\right)+\left(\frac{\mathbf{q} t-\mathbf{q}^{*}}{\mathbf{q}^{*}}\right)^{T} \mathbf{Q}_{m n}\left(\frac{\mathbf{k}_{t}-\mathbf{k}^{*}}{\mathbf{k}^{*}}\right) \\
+\rho\left(r_{t}-r^{*}\right)^{2}
\end{array}\right] \\
=-\left(\begin{array}{c}
\frac{\mathbf{k}_{0}-\mathbf{k}^{*}}{\mathbf{k}^{*}} \\
\frac{\mathbf{q} 0-\mathbf{q}^{*}}{\mathbf{q}^{*}}
\end{array}\right)^{T} \mathbf{P}\left(\begin{array}{c}
\frac{\mathbf{k}_{0}-\mathbf{k}^{*}}{\mathbf{k}^{*}} \\
\frac{\mathbf{q}_{0}-\mathbf{q}^{*}}{\mathbf{q}^{*}}
\end{array}\right)
\end{gathered}
$$

The Central Bank loss function is subject to a closed loop dynamics including the feedback rule:

$$
\left(\begin{array}{c}
\mathbf{k}_{t+1} \\
{ }_{t} \mathbf{q}_{t+1}
\end{array}\right)=(\underbrace{\left(\begin{array}{cc}
\mathbf{A}_{n n} & \mathbf{A}_{n m} \\
\mathbf{A}_{m n} & \mathbf{A}_{m m}
\end{array}\right)}_{\mathbf{A}}+\underbrace{\left(\begin{array}{c}
\mathbf{B}_{n 1} \\
\mathbf{B}_{m 1}
\end{array}\right)}_{\mathbf{B}} \underbrace{\left(\begin{array}{ll}
\mathbf{F}_{1 n} & \mathbf{F}_{1 m}
\end{array}\right)}_{-\mathbf{F}})\left(\begin{array}{c}
\mathbf{k}_{t} \\
\mathbf{q}_{t}
\end{array}\right)+\gamma \mathbf{z}_{t}
$$

where $\beta^{\prime}$ is a discount factor, $\mathbf{k}_{t}$ is an $(n \times 1)$ vector of variables predetermined at $t$ with initial conditions $\mathbf{k}_{0}$ given (shocks can straightforwardly be included into this vector); $\mathbf{q}$ is an $(m \times 1)$ vector of variables non-predetermined at $t ; \mathbf{z}$ is an $(k \times 1)$ vector of exogenous variables; $r$ is the policy interest rate, with a linear policy feedback rule $\mathbf{- F}$ which is a $1 \times(n+m)$ matrix; $\mathbf{Q}_{i j}$ is a $i \times j$ positive symmetric semi-definite matrix and $\rho>0$ is a scalar (both define the Central Bank preferences), $\mathbf{P}$ is a symmetric matrix (when $\mathbf{Q}$ is symmetric) which provides the optimal value of the loss function, $\mathbf{A}$ is $(n+m) \times(n+m)$ matrix, $\mathbf{B}$ is a $(n+m) \times 1$ matrix, $\gamma$ is a $(n+m) \times k$ matrix, ${ }_{t} \mathbf{q}_{t}$ is the agents expectations of $\mathbf{q}_{t+1}$ defined as follows:

$$
{ }_{t} \mathbf{q}_{t+1}=E_{t}\left(\mathbf{q}_{t+1}, \Omega_{t}\right) .
$$

$\Omega_{t}$ is the information set at date $t$ (it includes past and current values of all endogenous variables and may include future values of exogenous variables). According to Blanchard and Kahn (1980), a predetermined variable is a function only of variables known at date $t$ so that $\mathbf{k}_{t+1}={ }_{t} \mathbf{k}_{t+1}$ whatever the realization of the variables in $\Omega_{t+1}$. A non-predetermined variable can be 
a function of any variable in $\Omega_{t+1}$, so that we can conclude that $\mathbf{q}_{t+1}={ }_{t} \mathbf{q}_{t+1}$ only if the realization of all variables in $\Omega_{t+1}$ are equal to their expectations conditional on $\Omega_{t}$.

Boundary conditions for the policy-maker's first order conditions are the given initial conditions for predetermined variables $\mathbf{k}_{0}$ and Blanchard and Kahn (1980) hypothesis ruling out "bubbles", i.e. the exponential growth of the expectations of $\mathbf{w}=(\mathbf{k}, \mathbf{q}, \mathbf{z})$ :

$\forall t \in \mathbb{N}, \exists \overline{\mathbf{w}}_{t} \in \mathbb{R}^{k}, \exists \theta_{t} \in \mathbb{R}$, such that $\left|E_{t}\left(\mathbf{w}_{t+1} \mid \Omega_{t}\right)\right| \leq(1+i)^{\theta_{t}} \overline{\mathbf{w}}_{t}, \forall i \in \mathbb{R}^{+}$

Following Levine and Currie (1987) definition, a policy rule which imposes restrictions on the parameters of the rule is "quasi-optimal" in the sense that it is suboptimal in the general class of linear feedback rules but optimal within its own class. More precisely, the Central Bank is looking for such a quasi-optimal rule which imposes restrictions on the coefficients related to the non-predetermined variables. When the non-predetermined variables are excluded from the policy feedback rule, quasi-optimal rules are time consistent, because Calvo's (1978) shadow prices related to non predetermined variables (denoted $\mu_{\mathbf{q}}$ ) are no longer computed in the policymaker's optimization. These quasi-optimal rules lead naturally to a third type of rules: optimal time-consistent rules without pre-commitment related to discretionary policy where the policy-maker recursively optimizes again at each future period (Blake and Kirsanova (2012)).

Theorem 1 (Kwakernaak and Sivan (1972, p.198)). If the matrix pair $\left(\mathbf{A}_{n+m, n+m} \mathbf{B}_{n+m, 1}\right)$ is controllable, i.e. if the Kalman (1960) controllability matrix has full rank:

$$
\operatorname{rank}\left(\begin{array}{lllll}
\mathbf{B} & \mathbf{A B} & \mathbf{A}^{2} \mathbf{B} & \ldots & \mathbf{A}^{n+m-1} \mathbf{B}
\end{array}\right)=n+m
$$

the eigenvalues of $\mathbf{A}-\mathbf{B F}$ can be arbitrarily located in the complex plane (complex eigenvalues, however, occur in complex conjugate pairs) by choosing a policy rule matrix $\mathbf{F}$ accordingly.

The policy-maker considers only the set of policy rules $\mathbf{F}(n)$ such that $\mathbf{A}-\mathbf{B F}$ has exactly $n_{S}=n$ stable eigenvalues equal to the number of predetermined variables, in the hope to obtain Blanchard and Kahn (1980) unique rational expectations solution. Let $\mathbf{M}(\mathbf{F})$ be the matrix of left eigenvectors of $\mathbf{A}-\mathbf{B F}$ partitioned so that (indexes represent dimensions of the block matrices): 


$$
\begin{aligned}
& \left(\begin{array}{cc}
\mathbf{M}(\mathbf{F})_{n n} & \mathbf{M}(\mathbf{F})_{n m} \\
\mathbf{M}(\mathbf{F})_{m n} & \mathbf{M}(\mathbf{F})_{m m}
\end{array}\right)\left(\begin{array}{cc}
\mathbf{A}_{n n}-\mathbf{B}_{n 1} \mathbf{F}_{1 n} & \mathbf{A}_{n m}-\mathbf{B}_{n 1} \mathbf{F}_{1 m} \\
\mathbf{A}_{m n}-\mathbf{B}_{m 1} \mathbf{F}_{1 n} & \mathbf{A}_{m m}-\mathbf{B}_{m 1} \mathbf{F}_{1 m}
\end{array}\right) \\
& =\left(\begin{array}{cc}
\boldsymbol{\Lambda}_{n n} & \mathbf{0}_{n m} \\
\mathbf{0}_{m n} & \boldsymbol{\Lambda}_{m m}
\end{array}\right)\left(\begin{array}{cc}
\mathbf{M}(\mathbf{F})_{n n} & \mathbf{M}(\mathbf{F})_{n m} \\
\mathbf{M}(\mathbf{F})_{m n} & \mathbf{M}(\mathbf{F})_{m m}
\end{array}\right)
\end{aligned}
$$

where $\boldsymbol{\Lambda}_{n n}$ is a $n \times n$ diagonal matrix of stable roots, strictly lower than $1 / \sqrt{\beta}$ where $\beta$ is the discount factor of the policy maker, and $\boldsymbol{\Lambda}_{m m}$ is a $m \times m$ diagonal matrix of unstable roots. Then, the unique converging path is determined by a linear relationship between non-predetermined variables and predetermined variables (Blanchard and Kahn (1980)):

$$
\begin{aligned}
E_{t} \mathbf{q}_{t+1} & =-\mathbf{N}(\mathbf{F})_{m n} \mathbf{k}_{t+1}=-\mathbf{M}(\mathbf{F})_{m m}^{-1} \mathbf{M}(\mathbf{F})_{m n} \mathbf{k}_{t+1} \text { and } \\
\mathbf{q}_{0} & =-\mathbf{N}(\mathbf{F})_{m n} \mathbf{k}_{0}=-\mathbf{M}(\mathbf{F})_{m m}^{-1} \mathbf{M}(\mathbf{F})_{m n} \mathbf{k}_{0} .
\end{aligned}
$$

This describes "jumps" of non-predetermined variables to the stable manifold generated by predetermined variables. Then, the orthogonalized nonpredetermined variables (denoted $\mathbf{q}_{t}^{\prime}$ ) with unstable roots are linear combinations of orthogonalized predetermined variables with convergent eigenvalues (Blanchard and Kahn (1980) equation A6, p.1310). The formal derivation of matrix $\mathbf{N}(\mathbf{F})$ in the general case including stochastic shocks and when the generalized Schur method is necessary is presented in McCandless (2008), section 6.8 .

If the Central Bank defines a rule on both predetermined and non-predetermined variables $\left(\mathbf{F}_{1 n}, \mathbf{F}_{1 m}\right)$, this rule is observationally equivalent to a rule which depends only on predetermined variables with weights $\left(\mathbf{F}_{1 n}^{\prime}, \mathbf{0}_{1 m}\right)$ where the Central Bank imposes restrictions on the coefficients of the policy feedback rule, with all weights of non-predetermined variables equal to zero. This is detailed as follows:

$$
\begin{aligned}
r_{t}-r^{*} & =-\mathbf{F}_{1 n}\left(\frac{\mathbf{k}_{t+1}-\mathbf{k}^{*}}{\mathbf{k}^{*}}\right)-\mathbf{F}_{1 m}\left(\frac{\mathbf{q}_{t+1}-\mathbf{q}^{*}}{\mathbf{q}^{*}}\right) \\
& =-\underbrace{\left(\mathbf{F}_{1 n}-\mathbf{F}_{1 m} \mathbf{N}_{m n}\right)}_{\mathbf{F}_{1 n}^{\prime}}\left(\frac{\mathbf{k}_{t+1}-\mathbf{k}^{*}}{\mathbf{k}^{*}}\right), \\
\mathbf{F} & =\left(\mathbf{F}_{1 n}, \mathbf{F}_{1 m}\right)=\left(\mathbf{F}_{1 n}-\mathbf{F}_{1 m} \mathbf{N}_{m n}, \mathbf{0}_{1 m}\right) .
\end{aligned}
$$

The policy-maker only needs to control predetermined variables:

$$
\max _{\left\{R_{t}\right\}}-\frac{1}{2} \sum_{t=0}^{+\infty} \beta^{\prime t}\left[\mathbf{Q}_{n n}^{\prime}\left(\frac{\mathbf{k}_{t}-\mathbf{k}^{*}}{\mathbf{k}^{*}}\right)^{2}+\rho\left(r_{t}-r^{*}\right)^{2}\right]
$$


with a reduced loss function with weights $\mathbf{Q}_{n n}^{\prime}$ depending only on predetermined variables, which are observationally equivalent to the initial loss function depending on both non pre-determined variables and pre-determined variables according to the following equality:

$$
\mathbf{Q}_{n n}^{\prime}=\mathbf{Q}_{n n}+\mathbf{N}(\mathbf{F})_{n m}^{T} \mathbf{Q}_{m m} \mathbf{N}(\mathbf{F})_{m n}+\mathbf{Q}_{n m} \mathbf{N}(\mathbf{F})_{m n}+\mathbf{N}(\mathbf{F})_{n m}^{T} \mathbf{Q}_{m n}
$$

subject to the closed loop system of pre-determined variables:

$$
\mathbf{k}_{t+1}=\left(\mathbf{A}_{n n}^{\prime}-\mathbf{B}_{n 1} \mathbf{F}_{1 n}^{\prime}\right) \mathbf{k}_{t} .
$$

According to the following equality:

$$
\mathbf{A}_{n n}^{\prime}=\mathbf{A}_{n n}-\mathbf{A}_{n m} \mathbf{N}_{m n} \text { and } \mathbf{F}_{1 n}^{\prime}=\mathbf{F}_{1 n}-\mathbf{F}_{1 m} \mathbf{N}_{m n}
$$

the closed loop system of pre-determined variables is observationally equivalent to the top half of partitioned matrices of the non-controllable closed loop system including also non pre-determined variables:

$$
\begin{aligned}
\left(\begin{array}{c}
\mathbf{k}_{t+1} \\
t \mathbf{q}_{t+1}
\end{array}\right) & =\left(\begin{array}{cc}
\mathbf{A}_{n n}-\mathbf{B}_{n 1} \mathbf{F}_{1 n} & \mathbf{A}_{n m}-\mathbf{B}_{n 1} \mathbf{F}_{1 m} \\
\mathbf{A}_{m n}-\mathbf{B}_{m 1} \mathbf{F}_{1 n} & \mathbf{A}_{m m}-\mathbf{B}_{m 1} \mathbf{F}_{1 m}
\end{array}\right)\left(\begin{array}{c}
\mathbf{k}_{t} \\
\mathbf{q}_{t}
\end{array}\right) \text { with } \\
\left(\begin{array}{c}
\mathbf{k}_{t+1} \\
{ }_{t} \mathbf{q}_{t+1}
\end{array}\right) & =\left(\begin{array}{c}
\mathbf{I}_{n n} \\
-\mathbf{N}_{m n}
\end{array}\right)\left(\begin{array}{c}
\mathbf{k}_{t+1} \\
\mathbf{k}_{t+1}
\end{array}\right) \text { and }\left(\begin{array}{c}
\mathbf{k}_{t} \\
\mathbf{q}_{t}
\end{array}\right)=\left(\begin{array}{c}
\mathbf{I}_{n n} \\
-\mathbf{N}_{m n}
\end{array}\right)\left(\begin{array}{c}
\mathbf{k}_{t} \\
\mathbf{k}_{t}
\end{array}\right)
\end{aligned}
$$

The following proposition, initially formulated for optimal policy without pre-commitment by Blake and Kirsanova (2012), also holds for quasi-optimal policies with commitment:

Proposition 1: (Blake and Kirsanova (2012), p.1333). Let the closed loop transition matrix where the feedback policy rule $\mathbf{F}$ depends only on predetermined variables: $\mathbf{A}-\mathbf{B F}=\left(\begin{array}{cc}\mathbf{A}_{n n}-\mathbf{B}_{n 1} \mathbf{F}_{1 n} & \mathbf{A}_{n m} \\ \mathbf{A}_{m n}-\mathbf{B}_{m 1} \mathbf{F}_{1 n} & \mathbf{A}_{m m}\end{array}\right)$ have all distinct eigenvalues and be diagonalizable (which imply that the matrix pair $\left(\mathbf{A}_{n+m, n+m} \mathbf{B}_{n+m, 1}\right)$ is controllable). Let us consider the set of policy rules $\mathbf{F}\left(n_{S}\right)$ such that $\mathbf{A}-\mathbf{B F}$ has $n_{S}$ stable eigenvalues (below $1 / \sqrt{\beta}$ where $\beta$ is the discount factor of the policy maker) and $n-n_{S}$ unstable eigenvalues.

Case 1. For the set of policy rules $\mathbf{F}\left(n_{S}\right)$ such that $n_{S}<n$, the number of stable eigenvalues is strictly below the number of pre-determined variables, there is no rational expectations equilibrium according to Blanchard and Kahn (1980). 
Case 2. For the set of policy rules $\mathbf{F}\left(n_{S}\right)$ such that $n_{S}=n$, the number of stable eigenvalues is strictly equal to the number of predetermined variables, there is a unique rational expectations equilibrium according to Blanchard and Kahn (1980).

Case 3. For the set of policy rules $\mathbf{F}\left(n_{S}\right)$ such that $n<n_{S} \leq n+m$, there are at most $\frac{n_{S} !}{n !\left(n_{s}-n\right) !}$ ways of selecting $n$ stable eigenvalues and eigenvectors among a set of $n_{s}$ stable eigenvalues or eigenvectors, with parameters of the rule related to non-predetermined variables constrained to be equal to zero $\mathbf{F}_{1 m}=\mathbf{0}$. Hence, the matrix $\mathbf{N}\left(\mathbf{F}\left(n_{S}\right)\right)_{m n}$ based on these eigenvectors is not unique. It determines the linear relationship between non pre-determined variables to pre-determined variables:

$$
E_{t} \mathbf{q}_{t+1}=-\mathbf{N}(\mathbf{F})_{m n} \mathbf{k}_{t+1}=-\mathbf{M}(\mathbf{F})_{m m}^{-1} \mathbf{M}(\mathbf{F})_{m n} \mathbf{k}_{t+1}
$$

which are solutions of a particular non-symmetric Riccati matrix equation.

As emphasized by Blake and Kirsanova (2012), multiple equilibria may remain unnoticed. Choosing particular initial conditions for a DSGE may lead to convergence to a particular equilibrium using the currently available software programming Blanchard and Kahn (1980) solutions, without revealing the indeterminacy of the matrix $\mathbf{N}(\mathbf{F})_{m n}$.

Proposition 2. The following results hold for quasi-optimal rules if Blanchard and Kahn [1980] condition holds, that is, the number of non predetermined variables is equal to the number of unstable eigenvalues of the controlled system with the transition matrix $\mathbf{A}-\mathbf{B F}=\left(\begin{array}{cc}\mathbf{A}_{n n}-\mathbf{B}_{n 1} \mathbf{F}_{1 n} & \mathbf{A}_{n m} \\ \mathbf{A}_{m n}-\mathbf{B}_{m 1} \mathbf{F}_{1 n} & \mathbf{A}_{m m}\end{array}\right)$.

For a given matrix $\mathbf{N}_{m n}$ and if the matrix pair $\left(\mathbf{A}_{n n}^{\prime} \mathbf{B}_{n 1}^{\prime}\right)$ is controllable, i.e. if the following Kalman (1960) controllability matrix has full rank:

$$
\operatorname{rank}\left(\begin{array}{llllll}
\mathbf{B}_{n n}^{\prime} & \mathbf{A}_{n n}^{\prime} \mathbf{B}_{1 n}^{\prime} & \mathbf{A}_{n n}^{\prime 2} \mathbf{B}_{n 1}^{\prime} & \ldots & \mathbf{A}_{n n}^{\prime n-1} \mathbf{B}_{n 1}^{\prime}
\end{array}\right)=n
$$

the following results hold:

1. There is a unique symmetric positive semi-definite solution $\mathbf{P}$ to the discrete algebraic Ricatti equation:

$$
\mathbf{P}^{\prime}=\mathbf{Q}^{\prime}+\beta \mathbf{A}^{\prime T} \mathbf{P}^{\prime} \mathbf{A}^{\prime}-\beta \mathbf{A}^{\prime T} \mathbf{P}^{\prime} \mathbf{B}^{\prime}\left(\rho+\beta \mathbf{B}^{\prime T} \mathbf{P}^{\prime} \mathbf{B}^{\prime}\right)^{-1} \beta \mathbf{B}^{\prime T} \mathbf{P}^{\prime} \mathbf{A}^{\prime} .
$$

2. The restricted policy rule parameters of pre-determined variables $\mathbf{F}=\left(\mathbf{F}_{1 n}^{\prime}, \mathbf{0}_{1 m}\right)$ are uniquely determined from equation:

$$
\mathbf{F}_{1 n}^{\prime}=\beta\left(\rho+\mathbf{B}^{\prime T} \mathbf{P}^{\prime} \mathbf{B}^{\prime}\right)^{-1} \mathbf{B}^{\prime T} \mathbf{P}^{\prime} \mathbf{A}^{\prime} .
$$


3. Non identification of quasi-optimal rule parameters of non pre-determined variables (such as macro-prudential risk variables) if ever those rule parameters are distinct from zero:

$$
\mathbf{F}=\left(\mathbf{F}_{1 n}, \mathbf{F}_{1 m}\right)=\left(\mathbf{F}_{1 n}-\mathbf{F}_{1 m} \mathbf{N}_{m n}, \mathbf{0}_{1 m}\right) .
$$

4. Indeterminacy. According to proposition 1, there may be at most $\frac{(n+m) !}{n ! m !}$ multiple equilibria providing a matrix $\mathbf{N}(\mathbf{F})_{m n}$ :

$$
E_{t} \mathbf{q}_{t+1}=-\mathbf{N}(\mathbf{F})_{m n} \mathbf{k}_{t+1} \text { and } E_{t} \mathbf{q}_{0}=-\mathbf{N}(\mathbf{F})_{m n} \mathbf{k}_{0}
$$

5. Bounded solution. For the optimal policy rule $\mathbf{F}_{1 n}^{\prime}$ all eigenvalues of the closed loop transition matrix $\mathbf{A}_{n n}^{\prime}-\mathbf{B}_{n 1} \mathbf{F}_{1 n}^{\prime}$ are strictly less than $1 / \sqrt{\beta}$

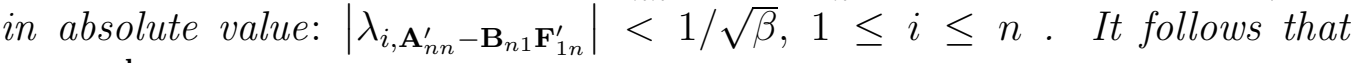
$\lim _{t \rightarrow+\infty} \frac{\mathbf{k}_{t}}{(\sqrt{\beta})^{t}}$. Then, non-predetermined variables are bounded because ${ }_{t} \mathbf{q}_{t+1}=$ $-\mathbf{N}_{m n} \mathbf{k}_{t+1}$.

6. Minimal volatility of the policy interest rate $(\rho>0, \mathbf{Q}=\mathbf{0})$. In this case, the policy rule parameters are all equal to zero. All the (stable) eigenvalues of the open loop system $\mathbf{A}_{n n}^{\prime}$ remain unchanged in the closed loop system $\left|\lambda_{i, \mathbf{A}-\mathbf{B F}}\right|=\left|\lambda_{i, \mathbf{A}}\right|<1$ (Rojas (2011)).

7. Inability of the policies to change the covariances matrix between predetermined and non pre-determined variables when $\mathbf{Q}_{m n} \neq \mathbf{0}$ and $\mathbf{Q}_{n m} \neq \mathbf{0}$, as it is fixed according to Blanchard and Khan (1980) condition: $E_{t} \mathbf{q}_{t+1}=$ $-\mathbf{N}(\mathbf{F})_{m n} \mathbf{k}_{t+1}$.

8. Time consistency à la Calvo (1978). As the quasi-optimal policy rule excludes non-predetermined variables from the optimal control problem, the Lagrange multipliers related to there variables (at the origin of time inconsistency issues) do not show up in the optimization.

Most of recent macro-prudential DSGE models assume simultaneously (1) ad hoc Taylor rules augmented by non pre-determined variables such as asset prices and private credit and compares them with non augmented Taylor rules, (2) no asset price bubbles and no Ponzi game condition for credit and asset prices with Blanchard and Kahn (1980) condition. These macroprudential DSGE models face the same identification problem as "quasioptimal" rules with pre-commitment. When analyzing the identification of all DSGE parameters and not only the rule parameters, the controllability hypothesis is also instrumental in several demonstrations in Komunjer and $\mathrm{Ng}$ (2011) appendix. Rule parameters are usually found to be in the list of least identified parameters for specific DSGE identification analysis, while autoregressive components of shocks are in the list of best identified parameters, see e.g. Caglar, Chadha and Shibayama (2012). 


\section{Identification and "over stable" rules with Central Bank pre-commitment to financial stability}

Proposition 3 summarizes the identification, determinacy and stability properties of "over stable" rational expectations optimal rules (Levine and Currie (1987) terminology) with Central Bank pre-commitment to financial stability, where the number of stable eigenvalues is larger than the number of pre-determined variables. Woodford's (2003) famous paper on Central Bank interest smoothing rules are "over stable" rational expectations optimal policy interest rate rules under commitment.

Ljungqvist and Sargent (2012, chapter 19) describe a four step algorithm for solving the optimal policy under commitment. "Step 1 seems to disregard the forward looking aspect of the problem. If we temporarily ignore the fact that the $\mathbf{q}_{0}$ component of the state $\mathbf{y}_{0}=\left(\frac{\frac{\mathbf{k}_{0}-\mathbf{k}^{*}}{\mathbf{k}^{*}}}{\frac{\mathbf{q}_{0}-\mathbf{q}^{*}}{\mathbf{q}^{*}}}\right)$ is not actually a state vector, then superficially the Stackelberg problem has the form of an optimal linear regulator problem" (Ljungqvist and Sargent (2012, chapter 19, p.769). Step one obtains the matrix $\mathbf{P}$ giving the optimal value of the loss function as a solution a matrix Riccati equation and the optimal parameters of the feedback rule $\mathbf{F}$ " as if" $\mathbf{q}_{t}$ are pre-determined variables. Step 2 seeks an "over stable" solution, that is a stabilizing solution for the $\mathbf{y}_{t}$ including all non-predetermined variables:

$$
\sum_{t=0}^{+\infty} \beta^{t} \mathbf{y}_{t}^{T} \mathbf{y}_{t}<+\infty
$$

solving the Lagrangian:

$$
-\frac{1}{2} \sum_{t=0}^{+\infty} \beta^{t}\left[\begin{array}{c}
\left(\frac{\mathbf{k}_{t}-\mathbf{k}^{*}}{\mathbf{k}^{*}}\right)^{T} \mathbf{Q}_{n n}\left(\frac{\mathbf{k}_{t}-\mathbf{k}^{*}}{\mathbf{k}^{*}}\right)+\left(\frac{\mathbf{q} t-\mathbf{q}^{*}}{\mathbf{q}^{*}}\right)^{T} \mathbf{Q}_{m m}\left(\frac{\mathbf{q}_{t}-\mathbf{q}^{*}}{\mathbf{q}^{*}}\right) \\
+\left(\frac{\mathbf{k}_{t}-\mathbf{k}^{*}}{\mathbf{k}^{*}}\right)^{T} \mathbf{Q}_{n m}\left(\frac{\mathbf{q}_{t}-\mathbf{q}^{*}}{\mathbf{q}^{*}}\right)+\left(\frac{\mathbf{q} t-\mathbf{q}^{*}}{\mathbf{q}^{*}}\right)^{T} \mathbf{Q}_{m n}\left(\frac{\mathbf{k}_{t}-\mathbf{k}^{*}}{\mathbf{k}^{*}}\right) \\
+\rho\left(r_{t}-r^{*}\right)^{2}+2 \beta \mu_{t+1}^{T}\left(\mathbf{A} \mathbf{y}_{t}+\mathbf{B}\left(r_{t}-r^{*}\right)-\mathbf{y}_{t+1}\right)
\end{array}\right]
$$

where $2 \beta^{\prime} \mu_{t+1}$ is the Lagrange multiplier associated with the linear constraint. First order conditions with respect to $r_{t}$ and $\mathbf{y}_{t}$, respectively, are:

$$
\begin{aligned}
0 & =\rho\left(r_{t}-r^{*}\right)+\beta \mathbf{B}^{T} \mu_{t+1} \\
\mu_{t} & =\mathbf{Q y}_{t}+\beta \mathbf{A}^{T} \mu_{t+1}
\end{aligned}
$$


Step 3 uses the property that a stabilizing solution satisfies:

$$
\mu_{t}=\left(\begin{array}{c}
\mu_{\mathbf{k}, t} \\
\mu_{\mathbf{q}, t}
\end{array}\right)=\mathbf{P}\left(\begin{array}{c}
\mathbf{k}_{t} \\
\mathbf{q}_{t}
\end{array}\right)=\left(\begin{array}{ll}
\mathbf{P}_{n n} & \mathbf{P}_{n m} \\
\mathbf{P}_{m n} & \mathbf{P}_{m m}
\end{array}\right)\left(\begin{array}{c}
\mathbf{k}_{t} \\
\mathbf{q}_{t}
\end{array}\right), \forall t \in \mathbb{N}
$$

Then, predetermined variables, the optimal policy rule $\boldsymbol{\Phi}$ and the closed loop system can be written as a function of predetermined variables $\left(\mathbf{k}_{t}, \mu_{\mathbf{q}, t}\right)$ :

$$
\begin{aligned}
\mathbf{q}_{t} & =\left(\begin{array}{cc}
-\mathbf{P}_{m m}^{-1} \mathbf{P}_{m n} & \mathbf{P}_{m m}^{-1}
\end{array}\right)\left(\begin{array}{c}
\mathbf{k}_{t} \\
\mu_{\mathbf{q}, t}
\end{array}\right) \text { and } \mathbf{q}_{0}=-\mathbf{P}_{m m}^{-1} \mathbf{P}_{m n} \mathbf{k}_{0} \text { if } \mu_{\mathbf{q}, t=0}=0 \\
r_{t} & =\mathbf{\Phi}\left(\begin{array}{c}
\mathbf{k}_{t} \\
\mu_{\mathbf{q}, t}
\end{array}\right)=-\mathbf{F}\left(\begin{array}{cc}
\mathbf{I}_{n n} & \mathbf{0}_{n m} \\
-\mathbf{P}_{m m}^{-1} \mathbf{P}_{m n} & \mathbf{P}_{m m}^{-1}
\end{array}\right)\left(\begin{array}{c}
\mathbf{k}_{t} \\
\mu_{\mathbf{q}, t}
\end{array}\right) \\
\left(\begin{array}{c}
\mathbf{k}_{t+1} \\
\mu_{\mathbf{q}, t+1}
\end{array}\right) & =\left(\begin{array}{cc}
\mathbf{I}_{n n} & \mathbf{0}_{n m} \\
\mathbf{P}_{m n} & \mathbf{P}_{m m}
\end{array}\right)(\mathbf{A}-\mathbf{B F})\left(\begin{array}{cc}
\mathbf{I}_{n n} & \mathbf{0}_{n m} \\
-\mathbf{P}_{m m}^{-1} \mathbf{P}_{m n} & \mathbf{P}_{m m}^{-1}
\end{array}\right)\left(\begin{array}{c}
\mathbf{k}_{t} \\
\mu_{\mathbf{q}, t}
\end{array}\right)
\end{aligned}
$$

To interpret empirical evidence about interest rate smoothing in the United States, Woodford (2003) eliminates the implementation Lagrange multipliers $\mu_{\mathbf{q}, t}$ in order to express the policy rule $\boldsymbol{\Phi}$ as a history-dependent representation of the policy rule denoted $\boldsymbol{\Psi}$ depending on the variables $\left(r_{t-1}, \mathbf{k}_{t}, \mathbf{k}_{t-1}\right)$.

Proposition 3. If the matrix pair $\left(\mathbf{A}_{n+m, n+m} \mathbf{B}_{n+m, 1}\right)$ is controllable, i.e. if the Kalman (1960) controllability matrix has full rank:

$$
\operatorname{rank}\left(\begin{array}{lllll}
\mathbf{B} & \mathbf{A B} & \mathbf{A}^{2} \mathbf{B} & \ldots & \mathbf{A}^{n+m-1} \mathbf{B}
\end{array}\right)=n+m
$$

the following results hold:

1. There is a unique symmetric positive semi-definite solution $\mathbf{P}$ to the discrete algebraic Ricatti equation:

$$
\mathbf{P}=\mathbf{Q}+\beta \mathbf{A}^{T} \mathbf{P A}-\beta \mathbf{A}^{T} \mathbf{P B}\left(\rho+\beta \mathbf{B}^{T} \mathbf{P B}\right)^{-1} \beta^{\prime} \mathbf{B}^{T} \mathbf{P A} .
$$

2. Uniqueness of the policy rule parameters of all controllable (pre-determined and non pre-determined) variables $\mathbf{F}$ which is determined from equation:

$$
\mathbf{F}=\beta\left(\rho+\mathbf{B}^{T} \mathbf{P B}\right)^{-1} \mathbf{B}^{T} \mathbf{P A} .
$$

3. Identification of optimal rule parameters. If the closed loop transition matrix $\mathbf{A}-\mathbf{B F}$ has all distinct eigenvalues denoted $\lambda_{i, \mathbf{A}-\mathbf{B F}}$ for $1 \leq i \leq$ $n+m$, there is a unique pole placement relationship between the set of distinct eigenvalues of the closed loop matrix $\lambda_{i, \mathbf{A}-\mathbf{B F}}$ and the unique solution of the set of parameters of the "as if $\mathbf{q}$ is predetermined" linear quadratic regulator policy rule $\mathbf{F}$. All coefficients of the "as if $\mathbf{q}$ is predetermined" policy rule 
$\mathbf{F}$ - related to both predetermined variables and non-predetermined variables - can be identified. Then, if $\mathbf{P}_{m m}$ is invertible, the parameters of rule $\mathbf{\Phi}$ applied on the pre-determined variables $\left(\mathbf{k}_{t}, \mu_{\mathbf{q}, t}\right)$ are also all identified. By contrast, the parameters of the history-dependent representation of the policy rule $\boldsymbol{\Psi}$ applied on the variables $\left(r_{t-1}, \mathbf{k}_{t}, \mathbf{k}_{t-1}\right)$ are usually not identified as their total number $2 n+1$ may differ from the number of distinct eigenvalues equal to the number of state variables $n+m$.

4. Determinacy. Kalman's controllability condition is a precondition for assuming that the Lagrange multipliers related to non predetermined variables should be all equal to zero at the initial date $\mu_{\mathbf{q}, t=0}=0$ (Bryson and Ho (1975), p.55-59; Xie (1997) provides a counter example where Kalman's controllability condition is not satisfied). As the Lagrange multipliers are related to the optimal value function matrix as follows: $\mu_{\mathbf{z}, t}=\mathbf{P}_{t}$, the initial values of non-predetermined variables are linear functions of the initial values of predetermined variables (Ljundqvist and Sargent's (2012, Chapter 19), Jensen (2011)):

$$
\mathbf{q}_{0}=-\mathbf{P}_{m m}^{-1} \mathbf{P}_{m n} \mathbf{k}_{0} \text { if } \mu_{\mathbf{q}, t=0}=0 .
$$

5. Bounded solution. For this policy rule $\mathbf{F}$ all eigenvalues of the closed loop transition matrix $\mathbf{A}-\mathbf{B F}$ (defining the evolution of the system under control) are strictly less than $1 / \sqrt{\beta}$ in absolute value. It follows that $\lim _{t \rightarrow+\infty} \frac{\mathbf{k}_{t}}{(\sqrt{\beta})^{t}}=\lim _{t \rightarrow+\infty} \frac{E_{t-1} \mathbf{q}_{t}}{(\sqrt{\beta})^{t}}=0$. Thus the policy reaction function ensures a finite loss and we may omit consideration of the Blanchard and Kahn's (1980) conditions on no bubbles on non-predetermined and predetermined variables (Levine and Currie's (1987) "over-stable" feedback rule and Woodford (2003)).

6. Minimal volatility of the policy interest rate $(\rho>0, \mathbf{Q}=\mathbf{0})$. It is such that stable eigenvalues of the open loop system are the same as in the closed loop system $\left|\lambda_{i, \mathbf{A}-\mathbf{B F}}\right|=\left|\lambda_{i, \mathbf{A}}\right|<1$ and that unstable eigenvalues (indexed by il) of the open loop system are mirrored by stable eigenvalues in the closed loop system having their modulus such that $\left|\lambda_{i^{\prime}, \mathbf{A}-\mathbf{B F}}\right|=1 /\left|\lambda_{i^{\prime}, \mathbf{A}}\right|<1$ (Rojas (2011)).

7. Ability of policies to decrease the covariances matrix between predetermined and non pre-determined variables when the policy maker preferences are such that $\mathbf{Q}_{m n} \neq \mathbf{0}$ and $\mathbf{Q}_{n m} \neq \mathbf{0}$.

8. Time inconsistency à la Calvo (1978). When the system is controllable and without a pre-commitment constraint, a policy maker who optimize again on period $t+1$ would choose an initial condition $\mu_{\mathbf{q}, t+1}=\mathbf{0}$ instead of the optimal path $\mu_{\mathbf{q}, t+1} \neq \mathbf{0}$ decided on date $t$. The system remains bounded and stable if ever the policy maker chooses $\mu_{\mathbf{q}, t+k}=\mathbf{0}$ on all following periods 
$(k>1)$ : the policy maker is not time inconsistent in the sense of promoting the instability of pre-determined variables, instead of their stability.

\section{Conclusion}

Parameters of financial instability variables in optimal policy rules with Central Bank commitment to financial stability can be identified, whereas it is not the case for "quasi-optimal" rules. Moreover, Kalman's (1960) controllability condition is a sufficient condition for determinacy and stability of these optimal policy rule under commitment.

Many extensions of optimal rules under commitment to financial stability are feasible. Firstly, all existing macro-prudential DSGE papers including an ad hoc augmented Taylor rules can be extended with additional simulations of optimal rules under commitment. These simulations and estimations would then be compared with the ones of ad hoc augmented Taylor rules. Secondly, robust optimal control à la Hansen and Sargent (2008), where the Central Bank optimal policy intends to minimize the worst of outcomes where they do not know with certainty fundamentals (as in Lorenzoni (2010)), is close to nowadays policy makers concerns.

Finally, the parameters of the Central Bank loss function should be endogenously determined. They result from a delegation problem taking into account the bargaining powers of the private sector divergent interests between lenders versus borrowers and between the banking sector versus nonfinancial sectors. Big banks benefit from financial instability over the business cycle through higher returns and larger informational rents during booms while being bailed out in case of distress. More precisely, the legal institutions surrounding the pre-commitment to a financial stability mandate should be investigated. Not only the Central Bank should be independent from government, but also it should be independent from the private banking sector.

\section{References}

[1] Anderson E.W., Hansen L.P., McGrattan E.R. and Sargent T.J. [1996]. Mechanics of Forming and Estimating Dynamic Linear Economies. in Amman H.M., Kendrick D.A. and Rust J. (editors) Handbook of Computational Economics, Elsevier, Amsterdam, 171-252. 
[2] Beau, D., L. CLerc, and B. Mojon [2012] "Macroprudential Policy and the Conduct of Monetary Policy." Bank of France Working Paper No. 390.

[3] Blake A.P. and Kirsanova T. [2012]. Discretionary Policy and Multiple Equilibria in LQ RE Models. Review of Economic Studies, 79, pp. 13091339 .

[4] Blanchard O.J. and Kahn C. [1980]. The solution of linear difference models under rational expectations. Econometrica, 48, pp. 1305-1311.

[5] Bryson A.E. and Ho Y.C. [1975]. Applied Optimal Control, John Wiley and Sons, New York.

[6] Burmeister E. [1980]. On some conceptual issues in rational expectations models. Journal of Money Credit and Banking, 12(4), pp. 800-16.

[7] Caglar E., Chadha J.S., Shibayama K. [2012]. Bayesian Estimation of DSGE models: Is the Workhorse Model Identified? EEA-ESEM conference.

[8] Calvo G.A. [1978]. On the Time Consistency of Optimal Policy in a Monetary Economy, Econometrica, 46(6), pp. 1411-1428.

[9] Chadha J.S., Corrado G. and Corrado L. [2013] Stabilisation Policy in a Model of Consumption, Housing Collateral and Bank Lending. Studies in Economics, 9, University of Kent school of economics discussion paper.

[10] Challe E. and Giannitsarou C. [2014]. Stock prices and monetary policy shocks: A general equilibrium approach. Journal of Economic Dynamics E Control, 40, pp. 46-66.

[11] Christiaans T. [2013]. Economic Crises, Housing Price bubbles and Saddle-Point Economics, Metroeconomica. 64(1), pp. 197-214.

[12] Cochrane J.H. [2011]. Determinacy and identification with Taylor Rules. Journal of Political Economy, 119(3), pp. 565-615.

[13] Komunjer I. and Ng S. [2011]. Dynamic Identification of Dynamic Stochastic General Equilibrium Models, Econometrica, 79(6), pp. 19952032 . 
[14] Gambacorta L. and Signoretti F.M. [2014]. Should monetary policy lean against the wind?: An analysis based on a DSGE model with banking. Journal of Economic Dynamics and Control. in Press.

[15] Hansen L.P. and Sargent [2008]. Robustness, Princeton University Press, Princeton.

[16] Jensen H. [2011]. Estimated Interest Rate Rules: Do they determine determinacy properties? The B.E. Journal of Macroeconomics, 11(1), pp 1-22.

[17] Kalman R.E. [1960]. Contributions to the Theory of Optimal Control. Boletin de la Sociedad Matematica Mexicana, 5, pp.102-109.

[18] Kwakernaak H. and Sivan R. [1972] Linear optimal control systems, Wiley, New York.

[19] Levine P. and Currie D. [1987]. The design of feedback rules in linear stochastic rational expectations models. Journal of Economic Dynamics and Control, 28, pages 1-28.

[20] Ljungqvist L. and Sargent T.J. [2012]. Recursive Macroeconomic Theory. 3rd edition. The MIT Press. Cambridge, Massaschussets.

[21] Loisel O. [2009]. Bubble-free policy feedback rules. Journal of Economic Theory, 144, pp. 1521-1559.

[22] Lorenzoni G. [2010]. Optimal Monetary Policy with Uncertain Fundamentals and Dispersed Information. Review of Economic Studies, 77, pp. 305-338.

[23] McCandless G.T. [2008]. The ABCs of RBCs: an introduction to dynamic macroeconomic models. Harvard University Press, Cambrigde, Massachusetts.

[24] Miller M. and Salmon M. [1985], Dynamic Games and the Time Inconsistency of Optimal Policy in Open Economies, Economic Journal, Vol. 95, Supplement: Conference Papers, pp. 124-137.

[25] Rojas A.J. [2011]. On the Discrete-Time Algebraic Riccati equation and its solution in closed-form. Proceedings of the 18th IFAC World congress, pp. 162-167. 
[26] Stein J.C. [2014]. Incorporating Financial Stability Considerations into a Monetary Policy Framework. Speeches of Federal Reserve Officials: International Research Forum on Monetary Policy, 21st March.

[27] Smets F. [2013]. Financial Stability and Monetary Policy: How Closely Interlinked? Sveriges Riksbank Economic Review. pp.121-160.

[28] Woodford M. [2003]. Optimal Interest-Rate Smoothing. Review of Economic Studies. 70, pp. 861-886.

[29] Xie D. [1997]. On time consistency: a technical issue in Stackelberg differential games. Journal of Economic Theory. 76, pp. 412-430. 